\title{
Enfermidades diagnosticadas em bezerros na região sul do Rio Grande do Sul ${ }^{1}$
}

\author{
Nathalia D. Assis-Brasil ${ }^{2}$, Clairton Marcolongo-Pereira², Fabiane L. Hinnah ${ }^{3}$, \\ Silvia R.L. Ladeira ${ }^{4}$, Eliza S.V. Sallis ${ }^{5}$, Fabiane B. Grecco ${ }^{5}$ e Ana Lucia Schild ${ }^{4 *}$
}

\begin{abstract}
Assis-Brasil N.D., Marcolongo-Pereira C., Hinnah F.L., Ladeira S.R.L., Sallis E.S.V., Grecco F.B. \& Schild A.L. 2013. [Diseases diagnosed in calves in southern Rio Grande do Sul, Brazil.] Enfermidades diagnosticadas em bezerros na região sul do Rio Grande do Sul. Pesquisa Veterinária Brasileira 33(4):423-430. Laboratório Regional de Diagnóstico, Faculdade de Veterinária, Universidade Federal de Pelotas, Campus Universitário s/n, Pelotas, RS 96010-900, Brazil. E-mail: alschild@terra.com.br

The aim of this study was to report the frequency of diseases affecting cattle under one year of age in the area of influence of the Regional Diagnostic Laboratory (LRD) of the Veterinary School of the Federal University of Pelotas (UFPel), establishing the main epidemiological factors associated with the occurrence of these diseases. The necropsy protocols and protocols of mailed in tissues from cattle under one year of age, submitted during 20002011 to LRD/UFPel for diagnosis, were reviewed. In 35.6\% of the cases, the calves were of dairy breeds, $33.9 \%$ were beef calves, $18.3 \%$ were of mixed breed, and in $12.1 \%$ of the cases the breed was not informed. The organ systems most affected were the central nervous system $(22.7 \%)$, digestive tract $(18.6 \%)$ and respiratory system (16.8\%). The diagnoses were divided by age groups: 88 calves were 1-90 days of age, 42 were 4-6 months, 32 were 7-9 months, and 44 cattle were 10-12 months of age. The disease most often diagnosed in 1 to 90-day-old calves were pneumonia, malformations and encephalitis/meningoencephalitis, with $19.3 \%, 15.9 \%$ and $11.3 \%$ of cases respectively. In 4 to 6-month-old calves, pneumonia occurred in $16.5 \%$ of cases, and blackleg and diarrhea accounted for $7.1 \%$ of diagnoses each. In 7 to 9-month-old calves the most frequent diseases were pneumonia and tetanus with $9.3 \%$ of cases, and babesiosis and gastrointestinal parasitosis with $6.2 \%$ each. In 10 to 12 -month-old calves, infection by BoHV-5 represented $13.6 \%$ of cases, and pneumonia, rabies and parasitosis was observed in $9 \%$ of cases each. Based on the results of this study it can be concluded that infectious diseases related to the respiratory system were important causes of mortality in calves of all ages until 12 months in the area of influence of LRD and their occurrence may be influenced by environmental factors and by management. Encephalitis/meningoencephalitis were also important as cause of mortality in 3-month-old calves.
\end{abstract}

INDEX TERMS: Calves, calf mortality, epidemiology.

\footnotetext{
${ }^{1}$ Recebido em 29 de dezembro de 2012.

Aceito para publicação em 15 de fevereiro de 2013.

Parte da Dissertação de Mestrado da primeira autora.

${ }^{2}$ Pós-Graduandos em Veterinária, Faculdade de Veterinária, Universidade Federal de Pelotas (UFPel), Campus Universitário s/n, Pelotas, RS 96010-900, Brasil.

${ }^{3}$ Graduanda em Veterinária, Faculdade de Veterinária, UFPel, Pelotas, RS.

${ }^{4}$ Médicos Veterinários do Laboratório Regional de Diagnóstico, Faculdade de Veterinária, UFPel, Pelotas, RS. *Autor para correspondência: alschild@terra.com.br

${ }^{5}$ Docentes do Departamento de Patologia Animal, Faculdade de Veterinária, UFPel, Pelotas, RS.
}

RESUMO.- 0 objetivo deste estudo foi relatar a frequência das enfermidades que ocorrem em bovinos até um ano de idade na área de influência do Laboratório Regional de Diagnóstico (LRD) da Faculdade de Veterinária da Universidade Federal de Pelotas (UFPel) estabelecendo os principais fatores epidemiológicos associados à ocorrência dessas enfermidades. Foram revisados os protocolos de necropsias realizadas e de materiais de bovinos até um ano de idade, encaminhados ao LRD/UFPel entre 2000 e 2011. Em 35,6\% dos casos, os bezerros eram de raças leiteiras e em $33,98 \%$ eram animais de raças de corte, $18,3 \%$ dos 
bezerros não tinham raça definida e em 12,1\% dos casos não constava a raça no protocolo de necropsia. Os sistemas mais afetados foram o sistema nervoso central $(22,7 \%)$, o digestivo $(18,6 \%)$ e o respiratório $(16,8 \%)$. Os diagnósticos foram divididos por faixa etária sendo que 88 bezerros tinham 1-90 dias de idade; 42 casos corresponderam a animais de 4-6 meses; 32 casos corresponderam a bezerros com 7-9 meses e 44 eram bezerros com 10-12 meses de idade. As enfermidades mais frequentemente diagnosticadas nos bezerros de 1-90 dias foram pneumonias, malformações e encefalites/meningoencefalites com 19,3\%, $15,9 \%$ e $11,3 \%$ dos casos, respectivamente. Nos bezerros com 4-6 meses de idade, as pneumonias ocorreram em $16,5 \%$ dos casos e o carbúnculo sintomático e as enterites representaram 7,1\% dos diagnósticos cada. Nos bezerros de 7-9 meses, as enfermidades mais frequentes foram pneumonias e tétano com 9,3\% dos casos e babesiose e parasitoses gastrintestinais com 6,2\% cada. Nos bezerros de 10-12 meses a infecção por BoHV-5 representou 13,6\% dos casos e as pneumonias, a raiva e as parasitoses foram observadas em 9,\% dos casos cada. Com base nos resultados deste trabalho pode-se concluir que as doenças infecciosas relacionadas ao sistema respiratório foram importantes causas de mortalidade em bezerros de todas as faixas etárias na área de influência do LRD e sua ocorrência pode ser influenciada por fatores ambientais e pelo manejo. As encefalites/meningoencefalites foram também importantes como causa de mortalidade em bezerros até os três meses de idade.

TERMOS DE INDEXAÇÃO: Bezerros, mortalidade de bezerros, epidemiologia.

\section{INTRODUÇÃO}

Em bovinos os índices de mortalidade de até $5 \%$ entre o nascimento e os primeiros 3 meses de idade são considerados normais (Machado Neto et al. 2004). No entanto, a mortalidade de bezerros representa importante causa de prejuízos econômicos, principalmente na produção leiteira, podendo alcançar a média de $8,4 \%$ das perdas no pré-desmame nos Estados Unidos (Losinger \& Henrichs 1997). Além disso, tem sido mencionado que a mortalidade neonatal no mundo pode atingir índices até 64\% (Khan \& Khan 1991). Em bovinos de corte, nos Estados Unidos, a mortalidade de animais jovens é considerada, também, a maior causa de perdas econômicas (Larson \& Tyler 2005).

0 maior índice de mortalidade de bovinos apresenta-se na fase de cria, devido ao bezerro ter maior vulnerabilidade, principalmente no primeiro mês de vida (Gómez, 2008), sendo que $50 \%$ das perdas ocorrem nas primeiras 24 horas após o nascimento (Laster \& Gregory 1973) e, ao final do primeiro mês estes valores podem atingir até 84\% (Khan \& Khan 1991). Além disso, o bezerro tem um papel importante como incubador biológico e amplificador de agentes etiológicos, uma vez que exposto a uma baixa concentração desses agentes, geralmente apresenta sinais clínicos ocultos ou leves, porém disseminará grande quantidade de patógenos, causando uma contaminação ambiental considerável (Larson \& Tyler 2005).
Diversos estudos relatam as causas de mortalidade em bovinos jovens em outros países como o Canadá (Donkersgoed et al., 1990, Gagea et al. 2006a, 2006b, Booker et al. 2008, Waldner et al. 2010), Estados Unidos (Adegboye et al. 1995), Argentina (Morrell et al. 2008) e Noruega (Gulliksen et al. 2009). No Brasil há poucos relatos e levantamentos que mencionam as causas de mortalidade de bovinos até um ano de idade, principalmente de raças leiteiras (Neto et al, 2004). Uma das principais causas de morte em bezerros de raças leiteiras é atribuída a falhas na transferência da imunidade passiva (Feitosa et al. 2010) através do colostro, além disso, a criação dos bezerros com substitutivos do leite que não tem formulação adequada à manutenção e desenvolvimento dos mesmos, é prática comum em propriedades de produção de leite (Lorenz et al. 2011).

Dados do Laboratório Regional de Diagnóstico (LRD) da Faculdade de Veterinária da Universidade Federal de Pelotas (UFPel) mostram que em um período de 11 anos, do total de necropsias realizadas no laboratório ou materiais provenientes de necropsias realizadas a campo, 15,4\% eram bovinos de até um ano de idade, o que justifica a realização de um levantamento das enfermidade que ocorrem nesta faixa etária na região de influência do LRD/UFPel.

$\mathrm{O}$ objetivo deste estudo foi relatar as enfermidades que ocorrem em bezerros até um ano de idade na área de influência do LRD/UFPel, estabelecendo os principais fatores epidemiológicos associados à ocorrência dessas enfermidades.

\section{MATERIAL E MÉTODOS}

Foram revisados os protocolos de necropsias de bovinos até um ano de idade realizadas pelos técnicos do LRD/UFPel e de materiais de necropsias de bovinos realizadas no campo por veterinários que enviaram os órgãos para análise histológica entre janeiro de 2000 e dezembro de 2011. Desses protocolos foram resgatados os dados referentes à idade, ao sexo, à raça, à procedência e ao quadro clínico-patológico bem como o diagnóstico. Foram, também, obtidos os dados de culturas bacterianas realizadas nos casos de doenças causadas por estes agentes. Os diagnósticos foram agrupados em categorias de doença e por sistema afetado. As diferentes enfermidades foram, também, distribuídas por época do ano e por faixa etária de 1-90 dias (88 casos ou surtos), 4-6 meses (42 casos ou surtos), 7-9 meses (32 casos ou surtos) e de 10-12 meses (44 casos ou surtos). Os materiais remetidos sem alteração ou inadequados para diagnóstico conclusivo foram classificados a parte.

\section{RESULTADOS}

De um total de 1994 necropsias de bovinos realizadas no LRD/UFPel ou de materiais de necropsias realizadas a campo, encaminhados ao LRD para diagnóstico, entre janeiro de 2000 e dezembro de 2011, 306 corresponderam a bovinos até um ano de idade. Destes, 220 casos tinham diagnóstico conclusivo e 46 foram inconclusivos. No Quadro 1 são apresentados os diagnósticos agrupados nas diferentes categorias de enfermidades dos 220 casos com diagnóstico conclusivo. Quarenta casos, por não se enquadrarem em nenhuma das categorias de doenças diagnosticadas, foram descritos conforme o que segue: tecidos ou órgãos sem lesão de significado patológico (20 casos), cadáveres ou ór- 
Quadro 1. Enfermidades diagnosticadas em bovinos até um ano de idade no Laboratório Regional de Diagnóstico da Universidade Federal de Pelotas entre e 2000 e 2011

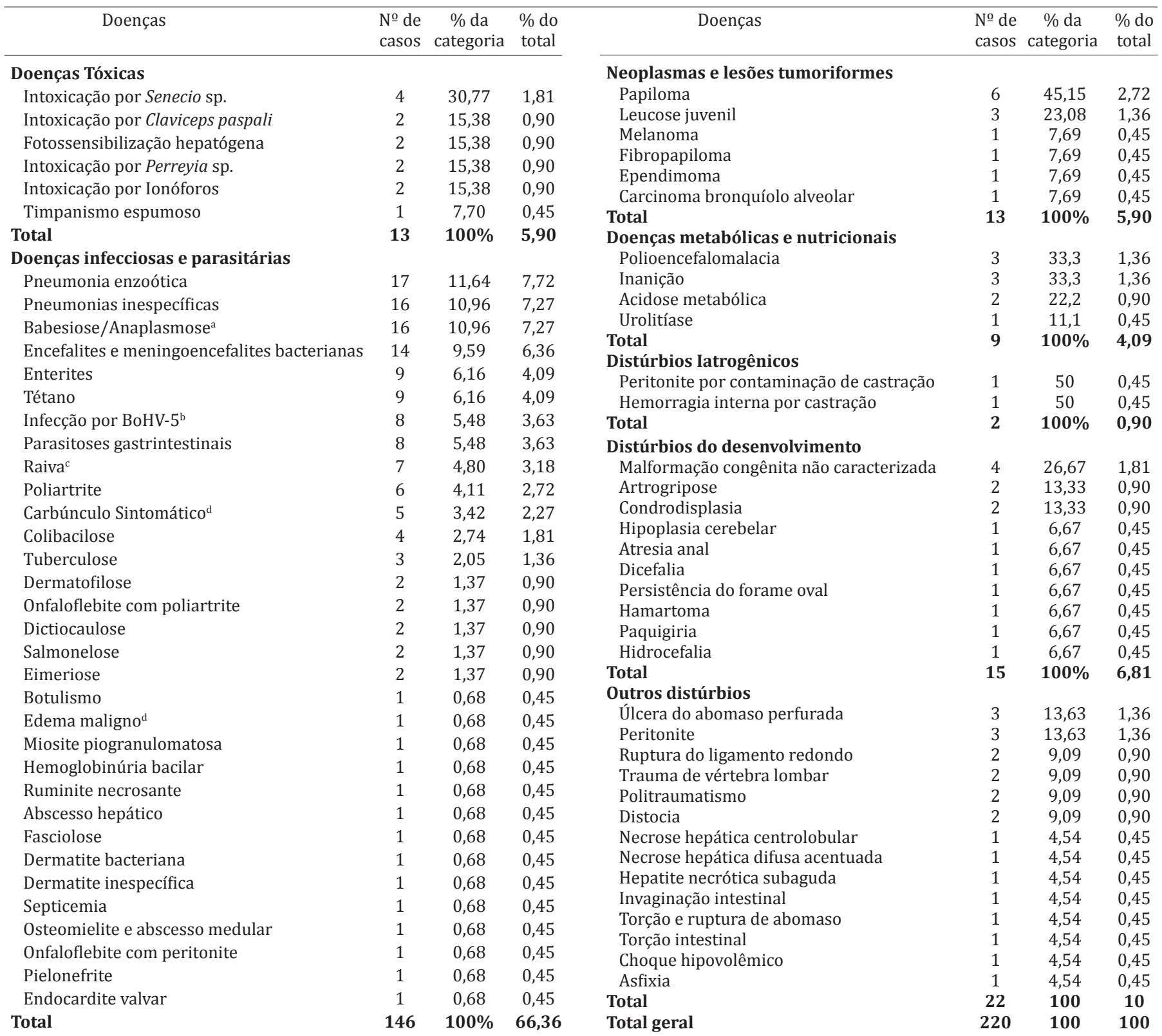

a Em dois casos não estava especificado o agente; ${ }^{\mathrm{b} B o} \mathrm{HV}-5$ = herpes vírus bovino- 5, diagnóstico realizado pela epidemiologia, sinais clínicos, lesões macro e microscópicas e presença de corpúsculos de inclusão intranucleares); ' diagnóstico realizado pelo IPVDF dentro do Programa Nacional de controle da raiva dos herbívoros (PNCRH) do Ministério da Agricultura Pecuária e Abastecimento; diagnóstico realizado pelas lesões características e pelo isolamento e caracterização de Clostridium presente nas lesões.

gãos em autólise avançada ( 6 casos), sangue de orelha ou cultura de medula óssea da tíbia negativos para carbúnculo hemático (4 casos), material inadequado para diagnóstico ( 4 casos), líquidos e exsudatos sem crescimento bacteriano ( 3 casos), sangue ou fragmentos de órgãos negativos para babesiose/anaplasmose (2 casos), e fragmentos de sistema nervoso central negativos para raiva (1 caso).

Dos 220 casos de bovinos com diagnóstico, 88 animais tinham 1-90 dias de idade, 42 casos corresponderam a bezerros de 4-6 meses, 32 casos corresponderam a bovinos com 7-9 meses e 44 eram bovinos com 10-12 meses de idade. Em 14 casos a idade não estava especificada no proto- colo, porém constava a categoria bezerro. No Quadro 2 são apresentados os casos distribuídos por sistema afetado. Os casos, quando possível, foram distribuídos, também, de acordo com o agente etiológico (Quadro 3).

Foram computadas as enfermidades mais frequentes observadas nas diferentes faixas etárias dos 206 casos com diagnóstico em que a idade dos bovinos afetados constava no protocolo (Quadro 4). Nos bovinos de 1-90 dias, as pneumonias representaram 19,3\% dos casos (17/88), seguidas pelas malformações com $15,9 \%$ dos casos $(14 / 88)$ (Fig.1), pelas encefalites/meningoencefalites com 11,3\% dos casos (10/88) (Fig.2 e 3) e pelas enterites com 5,6\% 
Quadro 2. Enfermidades diagnosticadas em bovinos até um ano de idade no Laboratório Regional de Diagnóstico da Universidade Federal de Pelotas entre 2000 e 2011, de acordo com os sistemas afetados

\begin{tabular}{lcc}
\hline \multicolumn{1}{c}{ Sistema } & N & $\%$ \\
\hline Sistema nervoso central & 50 & 22,72 \\
Digestivo & 41 & 18,63 \\
Sistema respiratório & 37 & 16,81 \\
Hemolinfático & 19 & 8,63 \\
Múltiplos sistemas & 18 & 8,18 \\
Glândulas anexas do sistema digestivo & 14 & 6,36 \\
Tegumentar & 13 & 5,90 \\
Ósseo e articular & 10 & 4,54 \\
Muscular & 9 & 4,09 \\
Outros & 4 & 1,81 \\
Cardiovascular & 3 & 1,36 \\
Urinário & 2 & 0,90 \\
Total & 220 & 100
\end{tabular}

Quadro 3. Enfermidades diagnosticadas em bovinos até um ano de idade no Laboratório Regional de Diagnóstico da Universidade Federal de Pelotas entre 2000 e 2011, de acordo com a etiologia

\begin{tabular}{lcc}
\hline \multicolumn{1}{c}{ Etiologia } & $\mathrm{N}$ & $\%$ \\
\hline Multifatorial & 45 & 20,45 \\
Bacteriana & 44 & 20,00 \\
Viral & 35 & 15,90 \\
Parasitária & 29 & 13,18 \\
Genética /ambiental $^{\mathrm{a}}$ & 15 & 6,81 \\
Tóxica $_{\text {Metabólica e nutricional }}$ & 13 & 5,90 \\
Neoplásica & 9 & 4,09 \\
Outros & 7 & 3,18 \\
Total & 23 & 10,45 \\
\hline
\end{tabular}

a Estão incluídos os defeitos congênitos que podem ser esporádicos ou ter origem genética ou ambiental.

(5/88) dos diagnósticos. Nos bovinos de 4-6 meses de idade as pneumonias representaram 16,5\% (7/42) dos diagnósticos, seguidas por carbúnculo sintomático e pelas enterites com 7,1\% (3/42) cada. Nos bovinos de 7-9 meses

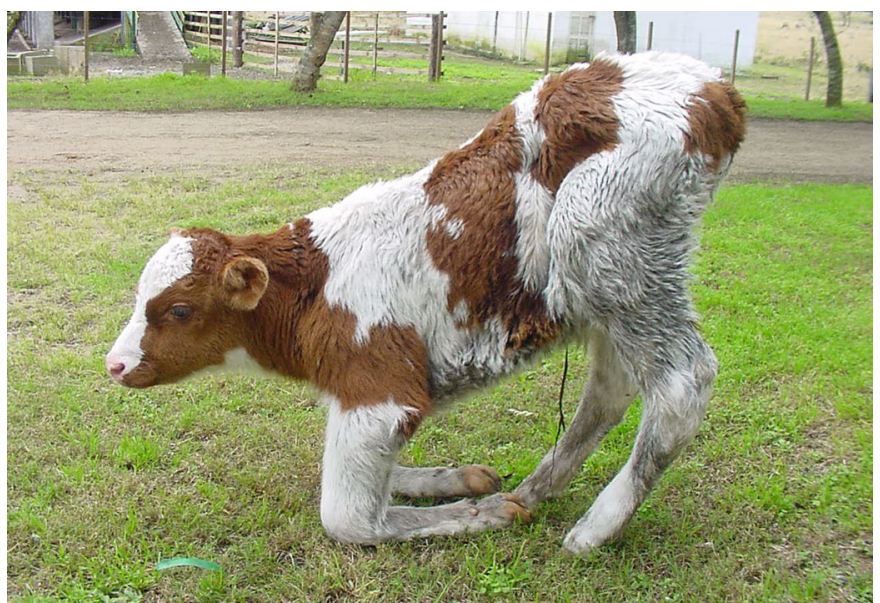

Fig.1. Bezerro recém nascido apresentando artrogripose dos membros anteriores que estão flexionados permanentemente.

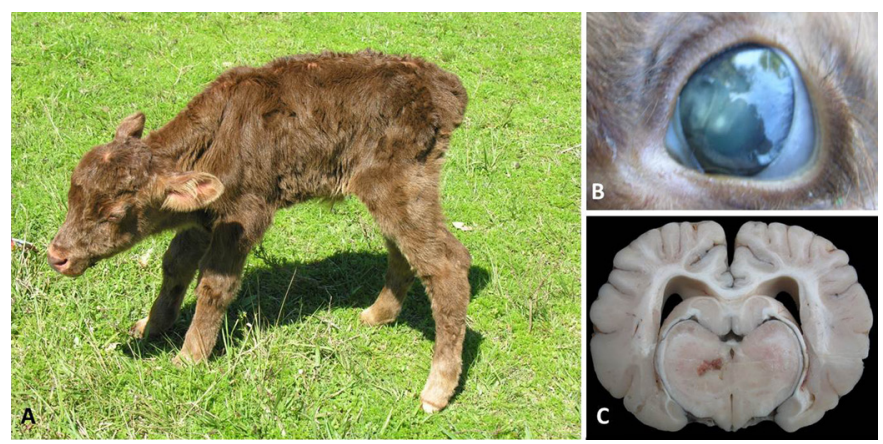

Fig.2. (A) Bezerro apresentando sinais clínicos neurológicos de depressão profunda. (B) Há cegueira evidenciada pela opacidade de córnea. (C) Presença de foco de hemorragia e necrose no sistema nervoso central na altura do tálamo.

as enfermidades mais frequentes foram as pneumonias e o tétano com $9,3 \%(3 / 32)$ cada, seguidas pela babesiose e por parasitoses gastrintestinais com 6,2\% (2/32) dos casos cada. Nos bovinos de 10-12 meses a infecção por

Quadro 4. Principais enfermidades diagnosticadas em bovinos até um ano de idade no Laboratório Regional de Diagnóstico da Universidade Federal de Pelotas entre 2000 e 2011, de acordo com a faixa etária

\begin{tabular}{|c|c|c|c|c|c|c|c|}
\hline Diagnóstico /1-90 dias & $\mathrm{N}$ & Diagnóstico/4-6 meses & $\mathrm{N}$ & Diagnósticos/7-9 meses & $\mathrm{N}$ & Diagnóstico/10-12 meses & $\mathrm{N}$ \\
\hline Pneumonias & 17 & Pneumonias & 7 & Pneumonias & 3 & Infecção por BoHV & 6 \\
\hline Malformações & 14 & Carbúnculo sintomático & 3 & Tétano & 3 & Pneumonias & 4 \\
\hline Encefalite/meningoencefalite & 10 & Enterites & 3 & Babesiose & 2 & Raiva & 4 \\
\hline Enterites & 5 & Infecção por BoHV- 5 & 2 & Parasitose gastrintestinal & 2 & Parasitose gastrintestinal & 4 \\
\hline Colibacilose & 4 & Babesiose & 2 & Polioencefalomalacia & 1 & Babesiose & 3 \\
\hline Salmonelose & 2 & Intoxicação por C. paspali & 2 & Intoxicação por Senecio sp. & 1 & Carbúnculo Sintomático & 2 \\
\hline Babesiose & 2 & Leucose juvenil & 1 & Raiva & 1 & Anaplasmose & 2 \\
\hline Leucose juvenil & 2 & Anaplasmose & 1 & Tuberculose & 1 & Tuberculose & 1 \\
\hline Tétano & 2 & Parasitose gastrointestinal & 1 & Outros diagnósticos & 18 & Intoxicação por Senecio sp. & 1 \\
\hline Anaplasmose & 1 & Dictiocaulose & 1 & Total & 32 & Tétano & 1 \\
\hline Poliencefalomalacia & 1 & Polioencefalomalacia & 1 & & & Intoxicação por P. flavipes ${ }^{c}$ & 1 \\
\hline Botulismo & 1 & Intoxicação por Senecio sp & 1 & & & Outros diagnósticos & 16 \\
\hline Eimeriose & 1 & Edema maligno & 1 & & & Total & 44 \\
\hline Dermatofilose & 1 & Tuberculose & 1 & & & & \\
\hline Intoxicação por P. flavipes & 1 & Tétano & 1 & & & & \\
\hline Raiva & 1 & Raiva & 1 & & & & \\
\hline Outros diagnósticos & 23 & Encefalites e meningites & 1 & & & & \\
\hline \multirow[t]{2}{*}{ Total } & 88 & Outros diagnósticos & 12 & & & & \\
\hline & & Total & 42 & & & & \\
\hline
\end{tabular}

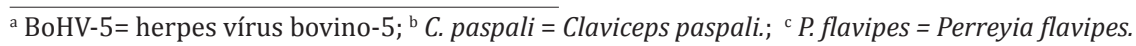



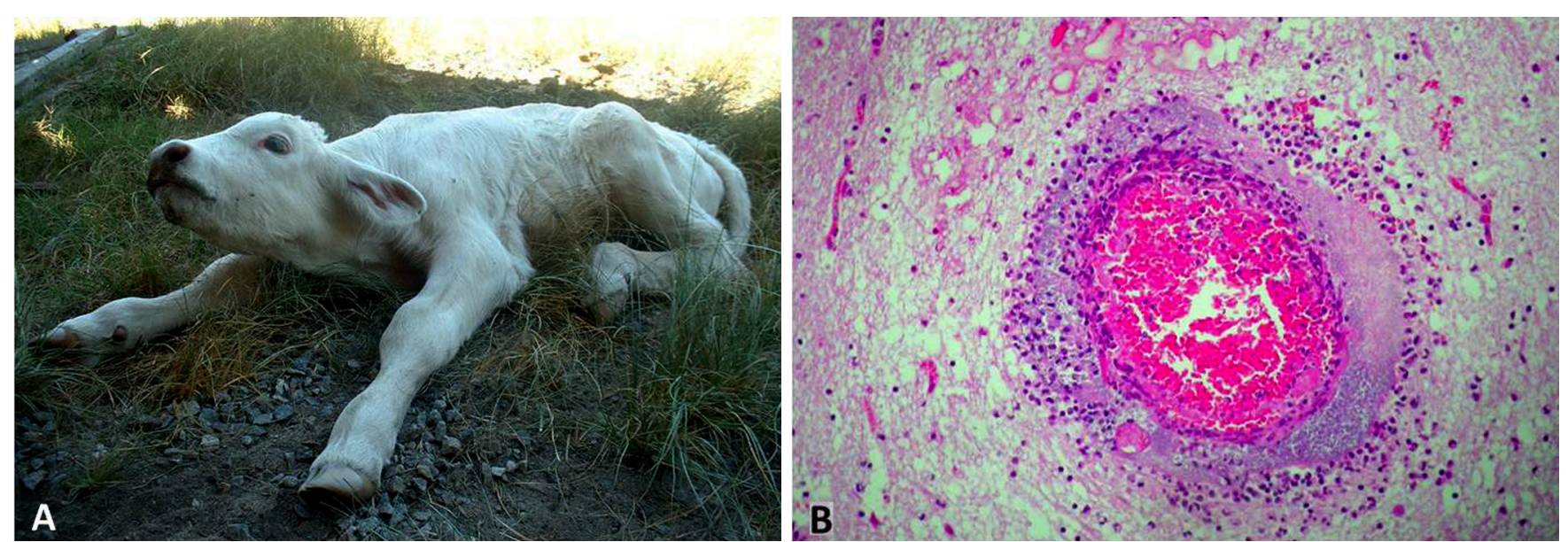

Fig.3. Meningoencefalite bacteriana. (A) Bezerro apresentando sinais neurológicos de opistótono sem conseguir manter-se em estação.

(B) Corte histológico do sistema nervoso central do bezerro com manguito perivascular de neutrófilos e presença de colônias bacterianas. HE, obj.20x.
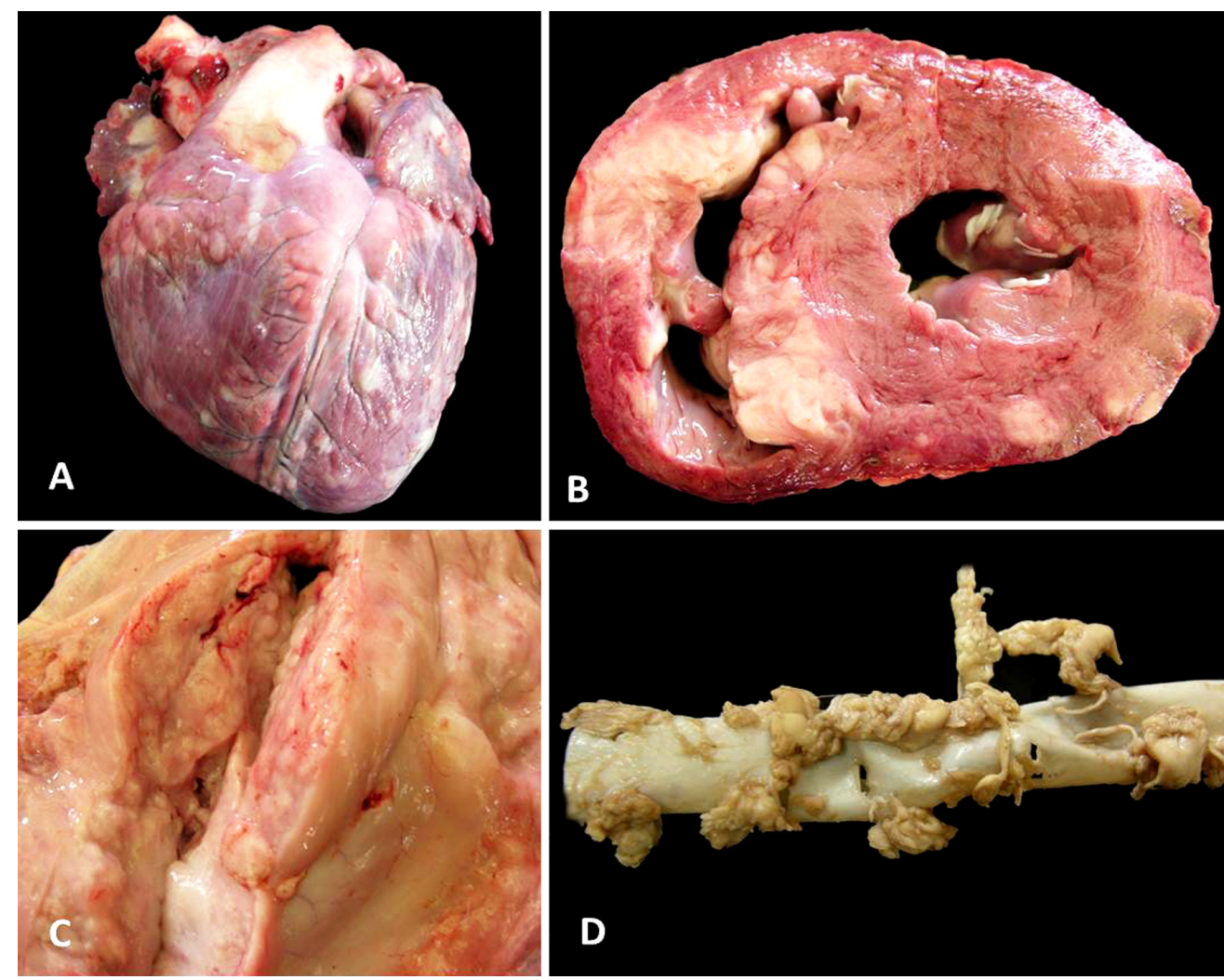

Fig.4. Leucose juvenil em bezerro de 2 meses de idade. (A) Há múltiplos nódulos esbranquiçados no epicárdio. (B) Miocárdio com áreas multifocais esbranquiçadas. (C) Abomaso com massa amarelada, homogênea e infiltrativa na parede do órgão. (D) Medula lombar com as raízes dos nervos periféricos recobertas por tecido amarelado e firme.

BoHV-5 representou $13,6 \%$ dos casos (6/44), seguida pelas pneumonias, pela raiva e parasitoses gastrintestinais com 9,\% (4/44) cada, babesiose com 6,8\% (3/44) e pelo carbúnculo sintomático e anaplasmose com 4,5\% (2/44) cada.

Dos 220 casos com diagnóstico, 10 corresponderam a biopsias das quais seis $(46,1 \%)$ tiveram o diagnóstico de papiloma, uma $(7,6 \%)$ de fibropapiloma, uma de melanoma, uma de hamartoma testicular e duas de dermatite.

Em 35,6\% (109/306) dos casos os bovinos afetados eram das raças leiterias, em 33,9\% (104/306) os animais afetados eram de raças de corte, em 18,30\% (56/306) dos casos os animais não tinham raça definida e em 12,09\% (37/306) dos casos não constava no protocolo de necropsia a raça do bovino.

Com relação à época de ocorrência das diferentes enfermidades observou-se que com exceção daquelas que são enfermidades estacionais, como babesiose e anaplasmose que ocorrem principalmente no verão e no outono e na intoxicação por Perreyia flavipes que ocorre no inverno, não 
houve diferença no número de casos/surtos com relação às estações do ano.

\section{DISCUSSÃO}

Os resultados do presente estudo demonstram que as enfermidades mais importantes que afetaram bovinos até um ano de idade na área de influência do LRD/UFPel no período de 2000-2011 estão relacionadas ao sistema nervoso central (SNC), ao sistema digestivo e ao sistema respiratório.

A categoria de enfermidade mais frequente em todas as faixas etárias foi a das doenças infecciosas e parasitárias representando mais da metade de todas as causas de morte observadas no período. Em outros estudos sobre mortalidade de bovinos menores de um ano de idade, agentes infecciosos como Histophilus somni (Donkersgoed et al. 1990), Mycoplasma bovis (Adegboye et al. 1995), vírus da diarreia viral bovina (BVDV) e agentes infecciosos que causam diarreia (Bendali et al. 1999) têm sido implicados como importantes causas de mortalidade. No presente estudo apesar de que em muitos casos não houve isolamento dos agentes infecciosos dentre os isolados destacaram-se as infecções por Escherichia coli, Clostridium chauvoei, Streptococcus spp., Salmonella sp., Pasteurella sp., Arcanobacterium pyogenes, Branhamella catarrhalis e Pseudomonas sp.

Com relação às enfermidades parasitárias observou-se que a babesiose foi a enfermidade mais frequente em bovinos a partir dos sete meses de idade. Isso está, provavelmente, relacionado à diminuição da imunidade passiva que os bezerros adquirem pelo colostro, a qual diminui após o desmame. Em um levantamento da ocorrência de tristeza parasitária bovina na área de influência do LRD/UFPel de 221 surtos diagnosticados nos anos de 1978-2005, 13,5\% ocorreram em bovinos menores de um ano (Almeida et al. 2006).

Neste estudo o número elevado de casos de doenças do SNC $(50 / 220)$ é pontual, uma vez que está incluída a raiva, cuja incidência aumentou na zona sul do Estado a partir do ano 2008. No período estudado em sete focos havia bovinos menores de um ano de idade afetados (Schild et al. 2012). Além disso, nos anos 2001 e 2009 numerosos surtos de tétano, que é uma enfermidade que se apresenta de forma esporádica, foram diagnosticados em todo Estado, o que elevou o número de casos incluídos como doenças deste sistema. Dois surtos de tétano entre os ocorridos em 2001 em consequência da administração de um medicamento contaminado (Driemeier et al. 2007), afetaram bovinos de até um ano. Os outros sete surtos foram diagnosticados em 2009, sendo sugerida uma condição epidemiológica específica para a ocorrência de grande número de surtos desta enfermidade no período (Quevedo et al. 2011).

No presente estudo foram diagnosticados 14 casos de meningoencefalite provavelmente de causa bacteriana sendo que houve crescimento de Eschericha coli em dois casos, Streptococcus sp. em um e bactérias contaminantes em outro. Meningite e encefalite bacterianas são importantes causas de morte de bezerros em outros países, incluindo a meningoencefalite trombótica causada por Histophilus somni (Benavides et al. 2011). Embora este agente não tenha sido isolado, lesões compatíveis com encefalite trom- bótica foram observadas em pelo menos um caso. Chama atenção que 10 dos 14 casos de meningoencefalite ocorreram em bezerros de até 90 dias de idade, sendo que em dois havia histórico de onfaloflebite logo após o nascimento. Em muitos casos as meningoencefalites estão relacionadas à infecção umbilical (Fernandes \& Schild 2007).

Oito surtos de meningoencefalite por BoHV-5 foram observados em bezerros a partir dos quatro meses até um ano de idade. Esta é uma enfermidade frequente na região sul do Estado e na mesma região nos anos de 1986-2003 foi observado que em 6 de 10 surtos os bovinos afetados tinham entre 2-12 meses de idade (Elias et al. 2004).

Estudos realizados em outros países como Canadá e Estados Unidos têm demonstrado que a mortalidade de bezerros está relacionada principalmente ao sistema respiratório (Gagea et al. 2006a, Snowder et al. 2006). No presente trabalho as enfermidades relacionadas ao sistema respiratório foram a terceira maior causa de mortalidade em animais até um ano de idade totalizando 33 surtos. No Canadá, mortalidade devido a enfermidades respiratórias foi observada em terneiros confinados maiores de dois meses de idade (Gagea et al. 2006b).

Não foi observada relação entre os surtos de pneumonias e as estações do ano. As pneumonias estão muito mais relacionadas ao estresse, a falhas na ingestão de colostro, ao baixo peso corporal e as condições higiênico-sanitárias e ambientais das instalações no caso de bezerros confinados do que propriamente com o clima, embora o frio também possa contribuir para a ocorrência da enfermidade (Driemeier \& Moojen 2007). Outros relatos mencionam, também, que não há diferença na mortalidade por pneumonia entre as diferentes estações do ano (Laster \& Gregory 1973).

Com relação ao sistema digestivo houve uma grande diversidade de enfermidades observadas perfazendo um total de 41 diagnósticos. Dentre esses casos destacaram-se as enfermidades que cursam com diarreia representando $60,9 \%$ dos casos (25/41). Tem sido mencionado que as principais causas de morte de bezerros até 30 dias são as diarreias que podem ser de etiologia viral, bacteriana, parasitária e alimentar (Botteon et al. 2008). A partir dos seis meses observou-se que as parasitoses por nematódeos gastrintestinais foram as principais enfermidades do trato digestivo. Bovinos são mais susceptíveis às parasitoses a partir do desmame, que na região estudada é realizado frequentemente nesta faixa etária (Ruas \& Berne 2007). No entanto, provavelmente o número de parasitoses diagnosticadas no presente estudo não reflete a realidade a campo uma vez que em muitos casos são remetidas fezes ao laboratório para diagnóstico e os animais são tratados, não havendo mortalidade.

As malformações congênitas foram frequentes e de modo geral observadas em animais da faixa etária até 90 dias. Esta frequência deve-se, provavelmente, ao fato de que proprietários e trabalhadores rurais em geral encaminham animais que nascem com malformações ao laboratório pela curiosidade que estes despertam. Estes casos geralmente são incompatíveis com a vida e os bezerros morrem pouco tempo após o nascimento (Leipold \& Dennis 1986). Apesar 
disso, um caso de hipoplasia cerebelar foi observado em um bovino de seis meses e um caso de paquigiria foi observado em um bovino de dois meses. Ambos os casos foram relatados anteriormente em um levantamento de defeitos congênitos realizados no LRD/UFPel (Marcolongo-Pereira et al. 2011). A sobrevivência destes animais além dos primeiros dias de vida aparentemente deve-se ao fato de ambas não serem lesões progressivas. Isto tem sido observado em algumas malformações congênitas do SNC (Summers et al. 1995).

Cabe ressaltar a ocorrência de três casos de leucose juvenil que é uma enfermidade tumoral de ocorrência esporádica descrita em bovinos principalmente com 3-6 meses de vida (Barros 2007). Deve ser considerado que neoplasmas observados na faixa-etária acima dos 6 meses têm importância relativa neste estudo, já que todos os casos, com exceção de um carcinoma bronquíolo-alveolar e de um ependimoma, eram provenientes de biopsias e não causaram a morte dos bovinos afetados.

Neste trabalho foi observado que as raças dos bezerros enviados ao LRD/UFPel para diagnóstico estiveram equilibradas entre as aptidões leiteira e de corte. A região sul do Estado caracteriza-se por criação extensiva de gado e também por uma bacia leiteira constituída por numerosas pequenas propriedades, o que justifica este equilíbrio.

Com base nos resultados deste trabalho pode-se concluir que as doenças infecciosas relacionadas ao sistema respiratório foram importantes causas de mortalidade em bezerros de todas as faixas etárias na área de influência do LRD e sua ocorrência pode ser influenciada por fatores ambientais e pelo manejo. As encefalites/meningoencefalites foram também importantes como causa de mortalidade em bovinos até os três meses de idade e uma das práticas para evitar sua ocorrência é a adequada desinfecção do umbigo logo após o nascimento.

\section{REFERÊNCIAS}

Almeida M.B., Tortelli F.P., Riet-Correa B., Ferreira J.L.M., Soares M.P., Farias N.A.R., Riet-Correa F. \& Schild A.L. 2006. Tristeza parasitária bovina na região sul do Rio Grande do Sul: estudo retrospectivo. Pesq. Vet. Bras. 26(4):237-242.

Adegboye D.S., Halbur P.G., Cavanaugh D.L., Werdin R.E., Chase C.C.L., Miskimins D.W. \& Rosenbusch R.F. 1995. Immunohistochemical and pathological study of Mycoplasma bovis-associated lung abscesses in calves. J. Vet. Diagn. Invest. 7:333-337.

Barros C.S.L. 2007. Leucose bovina, p.159-169. In: Riet-Correa F., Schild A.L., Lemos R.A.A. \& Borges J.R J. (Eds) Doenças de Ruminantes de Eqüídeos. Vol.2. 3a ed. Pallotti, Santa Maria.

Benavides J., Willoughby K., Underwood C., Newman B., Mitchell G. \& Carty H. 2011. Encephalitis and neuronal necrosis in a 3-month-old suckled beef calf. Vet. Pathol. 48(5):E1-E4. DOI: 10.1177/0300985810396515

Bendali F., Bichet H., Schelcher F. \& Sanaa M. 1999. Pattern of diarrhea in newborn beef calves in south-west France. Vet. Res. 30:61-74

Booker C.W., Abutarbush S.M., Morley P.S., Jim G.K., Pittman T.J., Schunicht O.C., Perrett T., Wildman B.K., Fenton R.K. \& Guichon P.T. 2008. Microbiological and histopathological findings in cases of fatal bovine respiratory disease of feedlot cattle in western Canada. Can. Vet. J. 49:473-481.

Botteon R.C.C., Botteon P.T.L., Santos Junior J.C.B., Pinna M.H. \& Lóss Z.G. 2008. Frequência de diarreia em bezerros mestiços sob diferentes condições de manejo na região do Médio Paraíba - Rio de Janeiro e Minas Gerais. Braz. J. Vet. Res. Anim. Sci. 45(2):153-160.
Donkersgoed J.V., Janzen E.D. \& Arland R.J. 1990. Epidemiological features of calf mortality due to hemophilosis in a large feedlot. Can. Vet. J. 31:821-825.

Driemeier D. \& Moojen V. 2007. Complexo respiratório bovino, p.490-496. In: Riet-Correa F., Schild A.L., Lemos R.A.A. \& Borges J.R J. (Eds), Doenças de Ruminantes de Eqüídeos. Vol.1. $3^{\text {a }}$ ed. Pallotti, Santa Maria.

Driemeier D., Schild A.L., Fernandes J.C.T., Colodel E.M., Corrêa A.M.R., Cruz C.E.F. \& Barros C.S.L. 2007. Outbreaks of tetanus in beef cattle and sheep in Brazil associated with disophenol injection. J. Vet. Med. A 54:333-335.

Elias F., Schild A.L. \& Riet-Correa F. 2004. Meningoencefalite e encefalomalacia por Herpesvirus bovino-5: distribuição das lesões no sistema nervoso central de bovinos naturalmente infectados. Pesq. Vet. Bras. 24(3):123-131.

Feitosa F.L.F., Camargo D.G., Yanaka R., Mendes L.C.N., Peiró J.R., Bovino F., Lisboa J.A.N., Perri S.H.V. \& Gasparelli E.R.F. 2010. Índices de falha de transferência de imunidade passiva (FTIP) em bezerros holandeses e nelores, às 24 e 48 horas de vida: valores de proteína total, de gamaglobulia, de imunoglobulina $\mathrm{G}$ e da atividade sérica de gamaglutamiltransferase, para o diagnóstico de FTIP. Pesq. Vet. Bras. 30(8):696-704.

Fernandes C.G. \& Schild A.L. 2007. Abscessos do sistema nervoso central, p.202-207. In: Riet-Correa F., Schild A.L., Lemos R.A.A. \& Borges J.R.J. (Eds), Doenças de Ruminantes de Eqüídeos. Vol.1. 3aㅡ ed. Pallotti, Santa Maria.

Gagea M.I., Bateman K.G., Dreumel T.V., McEwen B.J., Carman S., Archambault M., Shanahan R.A. \& Caswell J.L. 2006a. Diseases and pathogens associated with mortality in Ontario beef feedlots. J. Vet. Diagn. Invest. 18:18-28.

Gagea M.I., Kenneth G.B., Shanahan R.A., Dreumel T.V., McEwen B.J., Carman S., Archambault M., Shanahan R.A. \& Caswell J.L. 2006b. Naturally occurring Mycoplasma bovis-associated pneumonia and polyarthritis in feedlot beef calves. J. Vet. Diagn. Invest. 18:29-40.

Gómez R.G. 2008. Cria de becerras lecheras, p.43-68. In: Ibid. (Ed.), Enciclopedia Bovina. Universidad Nacional Autónoma de México, México.

Gulliksen S.M., Lie K.I., Loken T. \& Osteras O. 2009. Calf mortality in Norwegian dairy herds. J. Dairy Sci. 92:2782-2795. doi: 10.3168/jds.20081807.

Khan A. \& Khan M.Z. 1991. Aethiopathology of neonatal calf mortality. J. Islamic Acad. Sci. 4(2):159-165.

Larson R.L. \& Tyler J.W. 2005. Reducing calf losses in beef herds. Vet. Clin. Food Anim. Pract. 21:569-584.

Laster D.B. \& Gregory K.E. 1973. Factors influencing peri- and early postnatal calf mortality. J. Anim. Sci. 37(5):1092-1097.

Leipold H.W. \& Dennis S.M. 1986. Congenital defects affecting bovine reproduction, p.177-199. In: Morrow D.A. (Ed.), Current Therapy in Theriogenology: diagnosis, treatment and prevention of reproductive diseases in small and large animals. W.B. Saunders Company, Philadelphia.

Lorenz I., Mee J.F., Earley B. \& More S.J. 2011. Calf health from birth to weaning. I. General aspects of disease prevention. Irish Vet. J. 64(10):2-8.

Losinger W.C. \& Henrichs J. 1997. Management practices associated with high mortality among preweaned dairy heifers. J. Dairy Res. 64:1-11.

Machado Neto R., Faroni C.E., Pauletti P. \& Bessi R. 2004. Levantamento do manejo de bovinos leiteiros recém-nascidos: desempenho e aquisição de proteção passiva. Revta Bras. Zootec. 33(6, Supl.3):2323-2329.

Marcolongo-Pereira C., Schild A.L., Soares M.P., Vargas Jr S.F. \& Riet-Correa F. 2010. Defeitos congênitos diagnosticados em ruminantes na região Sul do Rio Grande do Sul. Pesq. Vet. Bras. 30:816-826.

Morrell E.L., Moore D.P., Odeón A.C., Poso M.A., Odriozola E., Cantón G., Paolicchi F., Malena R., Leunda M.R., Morsella C. \& Campero C.M. 2008. Retrospective study of bovine neonatal mortality: cases reported from INTA Balcarce, Argentina. Revta Argent. Microbiol. 40:151-157.

Quevedo P.S., Ladeira S.R.L., Soares M.P., Marcolongo-Pereira C., Sallis E.S.V., Grecco F.B., Estima-Silva P. \& Schild A.L. 2011. Tétano em bovinos no sul do Rio Grande do Sul. Pesq. Vet. Bras. 31:1066-1070.

Ruas J.L. \& Berne M.E.A. 2007. Parasitoses por nematódeos gastrintesti- 
nais em bovinos e ovinos, p.584-604. In: Riet-Correa F., Schild A.L., Lemos R.A.A. \& Borges J.R.J. (Eds), Doenças de Ruminantes de Eqüídeos. Vol.2. 3 a ed. Pallotti, Santa Maria.

Snowder G.D., Van Vleck L.D., Cundiff L.V. \& Bennett G.L. 2006. Bovine respiratory disease in feedlot cattle: environmental, genetic and economic factors. J. Anim. Sci. 84:1999-2008.

Schild A.L., Marcolongo-Pereira C., Adrien L.M., Fiss L., Ladeira S.R.L. \& Assis Brasil N.D. 2012. Doenças diagnosticadas pelo Laboratório Regional de Diagnóstico no ano 2011. Bolm Laboratório Regional de Diagnóstico 32, Editora e Gráfica Universitária, Pelotas. 53p.

Summers B.A., Cummings J.F. \& DeLahunta A. 1995. Veterinary Neuropathology. Mosby, Baltimore, p.300-327.

Waldner C.L., Kennedy R.I., Rosengren L.B., Pollock C.M. \& Clark E.G. 2010. Gross postmortem and histologic examination findings from abortion losses and calf mortalities in western Canadian beef herds. Can .Vet. J. 51:1227-1238. 